\title{
Measurement of degradation on under-utilized natural turf
}

\author{
Krisztina Varga - István Csízi \\ UD CAS Institutes for Agricultural Research and Educational Farm, Karcag 5300, Karcag, Kisújszállási út 166. \\ vargakrisztina@agr.unideb.hu
}

\begin{abstract}
SUMMARY
The role of turf serving animal husbandry is significantly declining with the decreasing number of grazing livestock in Hungary. Accordingly, the area of under-utilized or non-utilized turfs is increasing. At the University of Debrecen, Institutes for Agricultural Research and Educational Farm, Karcag Research Institute we studied four types of turf utilization in three repetitions on a salt meadow with Alopecurus pratensis. As a result of the performed examinations, we identified the composition of the flora structure on the investigated area and we measured carbon-dioxide circulation and soil moisture.
\end{abstract}

Keywords: under-utilized turf, Social Behaviour Types by Borhidi, coenological, carbon-dioxide emission, soil temperature, soil moisture

\section{INTRODUCTION}

Turf cultivation occupies $11 \%$ of agricultural area in our country (799.3 thousand ha) (Central Statistical Office, 2018), which should mean considerable forage base, but turf is the serving branch of grazing livestock and with the loss of its income and prestige this branch has gone into crisis (Vinczeffy, 1993). Beside these, the turf area in our country is gradually decreasing (Harcsa et al., 2008; 2009; 2011). Vinczeffy (1993) found that the natural, species-rich turfs is also decreasing because of tree plantations and building. More and more often extreme weather conditions also affect the turf culture (Halász et al., 2018). It is very important the fact that the turf uses 840 litre water for producing $1 \mathrm{~kg}$ dry matter without any nutrition (Barcsák et al., 1978; Szemán, 2006). Beside the decreasing tendency of turfs, most of our turfs are nature reservations (Molnár and Csízi, 2015). Because of this, so agrotechnical and utilization technologies must be applied in turf culture which can reach yield surplus and this can compensate yield loss caused by discarding nutrition resupply and irrigation (Dér et al., 2003; Bajnok et al., 2011).

Therefore, new concepts have been introduced into turf culture discipline (Szemán, 2006; Harcsa, 2009), these are the following: fertile turf, abandoned turf and fallow turf. Fertile turf is suitable for agricultural aims. The abandoned turf is at the beginning of the failure process. With the progress of succession, the fellow turf appears, where the valuable components of the turf are suppressed because of discarding utilization.

Yield of degraded turf is used, but because of inefficient methods the proportion of valuable turf components is gradually decreasing or missing, so the worthless species are dominant (Szente et al., 1998; Stefler et al., 2000; Magyar, 2009). The inefficient utilization has two types: over-utilization, in case of over-grazing load the turf becomes thinner and weeds avoided by livestock can spread in free places (Szente et al., 1998; Magyar, 2009; Czóbel et al., 2012). In case of under-utilization Penszka et al. $(2015 ; 2016)$ and Pápay (2016) wrote that succession processes endanger the life and survival of valuable turf components. Because of Williems and Bik, (1998), Török et al.
(2007) in European mountain turfs under-utilization is also a problem Stefler et al. (2000) and Halász (2018) confirmed that not only the overload, but the lack of utilization lead to turf degradation. According to Nagy (2001) on non-utilized areas the mother hay becomes old, sprouting is very moderate and the big size dicotyledonous weeds can dominate. Barcsák et al. (1978) state that flora components of non-utilized turfs change into an unfavourable direction, so the processes of natural succession boom, while Vinczeffy (1993) determined that because of the inefficient turf utilization these areas can become sea weed. Because of discarding to mow on the meadows reeds form (Szabó et al., 2010), on the dry turfs bushes and forest formation starts (Bajor et al., 2016; Hanson and Fogelfors, 2000; Kozák, 2011; Szentes et al., 2011; 2012). Erdös et al. (2013; 2014a; 2014b) found that with scrub formation species richness of turfs is decreasing. If the traditional turf treatment ceases, (Bakker and Berendse, 1999) on the under-utilized areas the amount of flammable grass moor will grow (Ryser et al., 1995), which increases the risk of grass fires (Brockway et al., 2006; Ónodi et al., 2008). Da Ronch et al. (2002) investigated the consequences of ceasing utilization and determined that the number flora species decreased to one fourth. Tóth et al. (2002) investigated the flora on bound soil, natural turfs which are similar to our experimental area and they observed that species richness decreases form grazing through mowing to zero utilization. According to Kahmen et al. (2002), for the adequate treatment the valuable species disappear on the area and at the same time the spread of competitor species threaten the survival of natural turfs (Isselstein et al., 2005). The consequence of the decreasing flora diversity is the poor fauna, too (Barcsák et al., 1978; Bartha et al., 2014). Furthermore, foreign species appear, which causes the ruin of the natural condition (Ferrer and Broca, 1999). Perevolotsky and Seligman (1998) state that the undergrazing leads to ,green desert". When the area becomes an impermeable bushy, the species richness of the area decreases and the danger of the wildfire increases on Mediterranean and dry areas because of water shortage. At the same time, Margóczi (2003) found that for 
preserving vegetation utilized and non-utilized areas are needed. According to Molnár and Csízi (2015), where there is pastored grazing method, the grazing from well to well can be a solution to terminate overgrazing near quarters and moor on far steppes. Nowadays, moor is a bigger problem on turfs than overgrazing because of decreasing number of grazing livestock. The dense grass moor blocks the germination of the valuable turf components, suppress the shorter species (fescues, Trifolium species), the worthless plants spread such as Elymus repens, and weeds can ripen seeds for instance Daucus carota, Cichorium intybus). In addition, on the under-utilized turfs the Glareola pratincola and the Alauda arvensis nestle, but their stocks are suppressed. In the past, moor turfs could be used because in spring grazing could be started earlier with using young grass growing on the moor turf from the previous year. The moor turf used as meadow was burnt with quick fire at the end of winter and a good meadow was gained (Baskay-Tóth, 1962).

\section{MATERIALS AND METHODS}

On the turf area number $01712 / 1$ of the University of Debrecen, Institutes for Agricultural Research and Educational Farm, Karcag investigations have been done since 2009. The area of the salt meadow with foxtails (Agrosti-Alopecuretum pratensis) belongs to the Natura2000 environmental regulations, so extensive meadow culture (one mowing a year, then grazing after grass) has been done since 1987. The area is $83 \mathrm{~m}$ above the sea level, the average amount of precipitation in the past 50 years is $503,4 \mathrm{~mm}$. In the investigation period, the total amount of precipitation was $353 \mathrm{~mm}$. The features of the months according to Vinczeffy (1993) climatic index were the following: in March desert (0.032), in April dry (0.122), in May a bit rainy (0.254), in June drought (0.095), in July drought (0.088), in August desert (0.020), and in September semi-desert (0.079) weather. The soil type is medium meadow solonec. At the beginning of the experiment we settled 4 treatments in 3 repetitions, the area of the parcels were net $30 \mathrm{~m}^{2}$ : „Ösgyep” (ancient lawn) zero treatment since 2009 (sign: A/Ö); „Mulcsozás” (mulching) stem-crushing in 3. decade of May since 2009 and mulch stays on the area (sign: A/M); „Egyoldalú kaszálás” (one-sided mowing) one mowing a year in 3. decade of May phytomass removal (sign: A/E); ,Legeltetett-Kontroll” (Grazing control) mowing in 3. decade of May, hay removal, grazing after grass in August with sheep (sign: A/L).

In our experiment we done the following methods: coenological monitoring, carbon-dioxide emission measurement, soil moisture measurement and soil temperature measurement.

Monitoring plants was done on $24^{\text {th }}$ May 2019 with the quadrate method by Balázs (Balázs, 1949). Its point is that we represented the occurrent plant species within the investigated quadrate or area with dominant value by Balázs $\left(D_{B}\right)$. After monitoring plant covering the plant species were classified into categories of Social Behaviour Types by Borhidi (Borhidi, 1993).
Measurement of carbon-dioxide emission, soil moisture and soil temperature were done every two weeks between $28^{\text {th }}$ March 2019 and $1^{\text {st }}$ October 2019. For measuring $\mathrm{CO}_{2}$ concentration, frame method developed by Research Institute Karcag was used with Testo 535 gas analyser. Measurement process is the following: after the impoundment of the area, it is covered, there is incubation time (30 min), then $\mathrm{CO}_{2}$ concentration is measured in the bailers (Kovács, 2014). For calculating $\mathrm{CO}_{2}$ emission values the following formula was used:

$$
\mathrm{F}=\mathrm{d} \times(\mathrm{V} / \mathrm{A}) \times\left(\mathrm{C}_{2}-\mathrm{C}_{1}\right) / \mathrm{t} \times 273 /(273+\mathrm{T})
$$

where: $\mathbf{F}=\mathrm{CO}_{2}$ emission $\left(\mathrm{kg} \times \mathrm{m}^{-2} \times \mathrm{h}^{-1}\right) ; \mathbf{d}=\mathrm{CO}_{2}$ volume mass $\left(1,96 \mathrm{~kg} \times \mathrm{m}^{-3}\right), \mathbf{V}=$ the barrel volume above soil surface $\left(0,0040 \mathrm{~m}^{3}\right), \mathbf{A}=$ measuring area $\left(0,0314 \mathrm{~m}^{2}\right)$, $\mathbf{C}_{1}=$ initial $\mathrm{CO}_{2}$ concentration $\left(\mathrm{m}^{3} \times \mathrm{m}^{-3}\right), \quad \mathbf{C}_{2}=\mathrm{CO}_{2}$ concentration after incubation $\left(\mathrm{m}^{3} \times \mathrm{m}^{-3}\right), \mathbf{t}=$ incubation time (1800 s), $\mathbf{T}=$ air temperature $\left({ }^{\circ} \mathrm{C}\right)$.

The measurement of $\mathrm{CO}_{2}$ emission depends on the moisture content and temperature of the soil and the air temperature, so at the same time of the measure we measured the air temperature, the moisture and temperature of the upper $10 \mathrm{~cm}$ layer of the soil. For measuring the moisture and the temperature of the soil, SMT-100 device was used, which measures dielectric conductivity of the soil in $0-10 \mathrm{~cm}$ deep and calculates the moisture in volume percentage. At the same time of this it measures the temperature of the layer, the results can be read on the screen of a hand data collector. The measurements were done in three repetitions. The data were analysed with descriptive statistic in Microsoft Office Excel.

\section{RESULTS AND DISCUSSION}

\section{Results of coenological observation and Social Behaviour Types by Borhidi (SBT) in 2019}

On the investigated area plant covering was monitored with quadrate method by Balázs $\left(\mathrm{D}_{\mathrm{B}}\right)$ and it was characterized with Social Behaviour Types categories by Borhidi, the results are shown in Table 1 .

In „Mulching” treatment Elymus repens, Vicia tetrasperma, Alopecurus pratensis and Poa pratensis occured in every three parcels. Rosa canina leans over form ancient lawn parcels. Uncovered areas occured in every three repetitions.

By One-sided mowing" treatment, Potentilla argentea, Vicia tetrasperma, Alopecurus pratensis and Poa pratensis occure in every three parcels. There are also uncovered areas in every three parcels.

By „Ancient lawn” treatment there is more, uncovered areas appear in every three parcels because of the spring drought. Alopecurus pratensis and Poa pratensis occur in every parcel of the treatment. Because of discarding mowing and succession processes bushes appeared on the area and Rosa canina spread (25-37.5\%). Our results proved findings of Szentes et al. (2011) and Bajor et al. (2016). Because of zero utilization the number of plant species strongly decreased, which proved Tóth et al. (2002). 
Coenological observation and Social Behaviour Types by Borhidi (SBT) in 2019 (Karcag)

\begin{tabular}{|c|c|c|c|c|c|}
\hline \multirow{2}{*}{ Parcel } & \multirow{2}{*}{ Plant species } & \multirow{2}{*}{$D_{B}$-value } & \multirow{2}{*}{ Covered area $(\%)$} & \multicolumn{2}{|c|}{ SBT by Borhidi } \\
\hline & & & & Sign & Value \\
\hline \multirow[t]{7}{*}{$\mathrm{A} / \mathrm{M} 1(1)$} & Uncovered area & 3 & 9.3750 & & \\
\hline & Potentilla argentea & 0.5 & 1.5625 & DT & 2 \\
\hline & Rosa canina & 1 & 3.1250 & DT & 2 \\
\hline & Elymus repens & 3 & 9.3750 & $\mathrm{RC}$ & -2 \\
\hline & Vicia tetrasperma & 2 & 6.2500 & DT & 2 \\
\hline & Alopecurus pratensis & 10 & 31.2500 & $\mathrm{C}$ & 5 \\
\hline & Poa pratensis & 12.5 & 39.0625 & G & 4 \\
\hline \multirow[t]{7}{*}{$\mathrm{A} / \mathrm{M} 2(2)$} & Uncovered area & 4 & 12.5000 & & \\
\hline & Potentilla argentea & 1 & 3.1250 & DT & 2 \\
\hline & Rosa canina & 1 & 3.1250 & DT & 2 \\
\hline & Elymus repens & 3 & 9.3750 & $\mathrm{RC}$ & -2 \\
\hline & Vicia tetrasperma & 1 & 3.1250 & DT & 2 \\
\hline & Alopecurus pratensis & 10 & 31.2500 & $\mathrm{C}$ & 5 \\
\hline & Poa pratensis & 12 & 37.5000 & $\mathrm{G}$ & 4 \\
\hline \multirow[t]{9}{*}{ A/M3(3) } & Uncovered area & 1 & 3.1250 & & \\
\hline & Silene alba & 0.5 & 1.5625 & $\mathrm{~W}$ & 1 \\
\hline & Elymus repens & 1 & 3.1250 & $\mathrm{RC}$ & -2 \\
\hline & Bromus pannonicus & 2 & 6.2500 & $\mathrm{C}$ & 5 \\
\hline & Cirsium arvense & 0.5 & 1.5625 & $\mathrm{RC}$ & -2 \\
\hline & Vicia tetrasperma & 0.5 & 1.5625 & DT & 2 \\
\hline & Galium aparine & 0.5 & 1.5625 & $\mathrm{~W}$ & 1 \\
\hline & Alopecurus pratensis & 10 & 31.2500 & $\mathrm{C}$ & 5 \\
\hline & Poa pratensis & 16 & 50.0000 & $\mathrm{G}$ & 4 \\
\hline \multirow[t]{8}{*}{$\mathrm{A} / \mathrm{E} 1(4)$} & Uncovered area & 1 & 3.1250 & & \\
\hline & Potentilla argentea & 1 & 3.1250 & DT & 2 \\
\hline & Elymus repens & 5 & 15.6250 & $\mathrm{RC}$ & -2 \\
\hline & Vicia tetrasperma & 1 & 3.1250 & DT & 2 \\
\hline & Alopecurus pratensis & 13 & 40.6250 & $\mathrm{C}$ & 5 \\
\hline & Poa pratensis & 10 & 31.2500 & $\mathrm{G}$ & 4 \\
\hline & Crepis setosa & 0.5 & 1.5625 & $\mathrm{~W}$ & 1 \\
\hline & Sonchus asper & 0.5 & 1.5625 & W & 1 \\
\hline \multirow[t]{9}{*}{$\mathrm{A} / \mathrm{E} 2(5)$} & Uncovered area & 2 & 6.2500 & & \\
\hline & Potentilla argentea & 1 & 3.1250 & DT & 2 \\
\hline & Silene alba & 1 & 3.1250 & $\mathrm{~W}$ & 1 \\
\hline & Vicia tetrasperma & 1 & 3.1250 & DT & 2 \\
\hline & Veronica persica & 1 & 3.1250 & $\mathrm{~W}$ & 1 \\
\hline & Taraxacum officinale & 1 & 3.1250 & $\mathrm{RC}$ & -2 \\
\hline & Alopecurus pratensis & 13 & 40.6250 & $\mathrm{C}$ & 5 \\
\hline & Poa pratensis & 11 & 34.3750 & G & 4 \\
\hline & Crepis setosa & 1 & 3.1250 & $\mathrm{~W}$ & 1 \\
\hline \multirow[t]{8}{*}{$\mathrm{A} / \mathrm{E} 3(6)$} & Uncovered area & 1.5 & 4.6875 & & \\
\hline & Potentilla argentea & 1 & 3.1250 & DT & 2 \\
\hline & Elymus repens & 6 & 18.7500 & $\mathrm{RC}$ & -2 \\
\hline & Cirsium arvense & 1 & 3.1250 & $\mathrm{RC}$ & -2 \\
\hline & Vicia tetrasperma & 1 & 3.1250 & DT & 2 \\
\hline & Alopecurus pratensis & 9 & 28.1250 & $\mathrm{C}$ & 5 \\
\hline & Poa pratensis & 12 & 37.5000 & $\mathrm{G}$ & 4 \\
\hline & Sonchus asper & 0.5 & 1.5625 & $\mathrm{~W}$ & 1 \\
\hline \multirow[t]{4}{*}{ A/Ö1(7) } & Uncovered area & 2 & 6.2500 & & \\
\hline & Rosa canina & 12 & 37.5000 & DT & 2 \\
\hline & Alopecurus pratensis & 5 & 15.6250 & $\mathrm{C}$ & 5 \\
\hline & Poa pratensis & 13 & 40.6250 & $\mathrm{G}$ & 4 \\
\hline \multirow[t]{7}{*}{ A/Ö2(8) } & Uncovered area & 0.5 & 1.5625 & & \\
\hline & Elymus repens & 1 & 3.1250 & $\mathrm{RC}$ & -2 \\
\hline & Cirsium arvense & 0.5 & 1.5625 & $\mathrm{RC}$ & -2 \\
\hline & Vicia tetrasperma & 0.5 & 1.5625 & DT & 2 \\
\hline & Galium aparine & 0.5 & 1.5625 & $\mathrm{~W}$ & 1 \\
\hline & Alopecurus pratensis & 28 & 87.5000 & $\mathrm{C}$ & 5 \\
\hline & Poa pratensis & 1 & 3.1250 & $\mathrm{G}$ & 4 \\
\hline
\end{tabular}


Table 1 continued

\begin{tabular}{|c|c|c|c|c|c|}
\hline \multirow{2}{*}{ Parcel } & \multirow{2}{*}{ Plant species } & \multirow{2}{*}{$D_{B}$-value } & \multirow{2}{*}{ Covered area $(\%)$} & \multicolumn{2}{|c|}{ SBT by Borhidi } \\
\hline & & & & Sign & Value \\
\hline \multirow[t]{6}{*}{ A/Ö3(9) } & Uncovered area & 1 & 3.1250 & & \\
\hline & Rosa canina & 8 & 25.0000 & DT & 2 \\
\hline & Elymus repens & 4 & 12.5000 & $\mathrm{RC}$ & -2 \\
\hline & Cirsium arvense & 0.5 & 1.5625 & $\mathrm{RC}$ & -2 \\
\hline & Alopecurus pratensis & 13.5 & 42.1875 & $\mathrm{C}$ & 5 \\
\hline & Poa pratensis & 5 & 15.6250 & $\mathrm{G}$ & 4 \\
\hline \multirow[t]{14}{*}{$\mathrm{AL} / 1(10)$} & Uncovered area & 2 & 6.2500 & & \\
\hline & Silene alba & 0.5 & 1.5625 & $\mathrm{~W}$ & 1 \\
\hline & Podospermum canum & 1 & 3.1250 & $\mathrm{G}$ & 4 \\
\hline & Cardaria draba & 0.5 & 1.5625 & W & 1 \\
\hline & Plantago lanceolata & 0.5 & 1.5625 & DT & 2 \\
\hline & Polygonum aviculare & 0.5 & 1.5625 & $\mathrm{RC}$ & -2 \\
\hline & Cirsium arvense & 0.5 & 1.5625 & $\mathrm{RC}$ & -2 \\
\hline & Vicia tetrasperma & 1 & 3.1250 & DT & 2 \\
\hline & Achillea setacea & 2 & 6.2500 & $\mathrm{G}$ & 4 \\
\hline & Ranunculus acris & 0.5 & 1.5625 & G & 4 \\
\hline & Alopecurus pratensis & 4 & 12.5000 & $\mathrm{C}$ & 5 \\
\hline & Inula britannica & 2 & 6.2500 & DT & 2 \\
\hline & Poa pratensis & 2 & 6.2500 & G & 4 \\
\hline & Festuca pseudovina & 15 & 46.8750 & $\mathrm{C}$ & 5 \\
\hline \multirow[t]{19}{*}{$\mathrm{AL} / 2(11)$} & Convolvulus arvensis & 0.5 & 1.5625 & $\mathrm{RC}$ & -2 \\
\hline & Uncovered area & 2 & 6.2500 & & \\
\hline & Plantago schwarzenbergiana & 0.5 & 1.5625 & $\mathrm{Sr}$ & 8 \\
\hline & Euphorbia cyparissias & 1 & 3.1250 & DT & 2 \\
\hline & Silene alba & 0.5 & 1.5625 & $\mathrm{~W}$ & 1 \\
\hline & Podospermuт сапит & 1 & 3.1250 & G & 4 \\
\hline & Elymus repens & 1 & 3.1250 & $\mathrm{RC}$ & -2 \\
\hline & Plantago lanceolata & 1 & 3.1250 & DT & 2 \\
\hline & Eryngium campestre & 1 & 3.1250 & DT & 2 \\
\hline & Lathyrus tuberosus & 0.5 & 1.5625 & W & 1 \\
\hline & Taraxacum officinale & 1 & 3.1250 & $\mathrm{RC}$ & -2 \\
\hline & Bromus hordeaceus & 1 & 3.1250 & DT & 2 \\
\hline & Achillea setacea & 1 & 3.1250 & $\mathrm{G}$ & 4 \\
\hline & Trifolium resutum & 0.5 & 1.5625 & $\mathrm{~S}$ & 6 \\
\hline & Alopecurus pratensis & 3 & 9.3750 & $\mathrm{C}$ & 5 \\
\hline & Cerastium vulgare & 0.5 & 1.5625 & DT & 2 \\
\hline & Inula britannica & 1 & 3.1250 & DT & 2 \\
\hline & Poa pratensis & 2 & 6.2500 & $\mathrm{G}$ & 4 \\
\hline & Festuca pseudovina & 13 & 40.6250 & $\mathrm{C}$ & 5 \\
\hline \multirow[t]{19}{*}{$\mathrm{AL} / 3(12)$} & Festuca rupicola & 2 & 6.2500 & $\mathrm{C}$ & 5 \\
\hline & Uncovered area & 1 & 3.1250 & & \\
\hline & Trifolium repens & 0.5 & 1.5625 & DT & 2 \\
\hline & Silene alba & 0.5 & 1.5625 & $\mathrm{~W}$ & 1 \\
\hline & Podospermuт сапит & 1 & 3.1250 & $\mathrm{G}$ & 4 \\
\hline & Elymus repens & 1 & 3.1250 & $\mathrm{RC}$ & -2 \\
\hline & Plantago lanceolata & 1 & 3.1250 & DT & 2 \\
\hline & Bromus pannonicus & 0.5 & 1.5625 & $\mathrm{C}$ & 5 \\
\hline & Cirsium arvense & 0.5 & 1.5625 & $\mathrm{RC}$ & -2 \\
\hline & Eryngium campestre & 0.5 & 1.5625 & DT & 2 \\
\hline & Bromus hordeaceus & 1 & 3.1250 & DT & 2 \\
\hline & Achillea setacea & 1 & 3.1250 & G & 4 \\
\hline & Trifolium resutum & 0.5 & 1.5625 & $\mathrm{~S}$ & 6 \\
\hline & Alopecurus pratensis & 5 & 15.6250 & $\mathrm{C}$ & 5 \\
\hline & Rumex obtusifolius & 0.5 & 1.5625 & DT & 2 \\
\hline & Inula britannica & 1 & 3.1250 & DT & 2 \\
\hline & Poa pratensis & 1 & 3.1250 & $\mathrm{G}$ & 4 \\
\hline & Festuca pseudovina & 13 & 40.6250 & $\mathrm{C}$ & 5 \\
\hline & Lotus corniculatus & 0.5 & 1.5625 & DT & 2 \\
\hline
\end{tabular}

1: „Mulching” treatment 1, 2: „Mulching” treatment 2, 3: „,Mulching” treatment 3, 4: „One-sided mowing” treatment 1, 5: „One-sided mowing” treatment 2, 6: „One-sided mowing” treatment 3, 7: „Ancient lawn” treatment 1, 8: „Ancient lawn” treatment 2, 9: „Ancient lawn” treatment 3, 10: „Grazing control” treatment 1, 11: „Grazing control” treatment 2, 12: „Grazing control” treatment 3. 
On „Grazing control” area lawn components were the most diverse. Podospermum canum), Plantago lanceolata, Achillea setacea, Alopecurus pratensis, Inula Britannica, Poa pratensis and Festuca pseudovina occure in every three repetitions. Uncovered areas occure in every three parcels.

Social behaviour types (SBT) of plant species based on their role in the association. SBT shows natural and disturbing state of the association between the plant and the habitat, from its proportion we can conclude natural state, regeneration and difference from natural state of the association (Borhidi, 1993).

In our study, Festuca rupicola, Bromus pannonicus, Alopecurus pratensis and Festuca pseudvina were classified into the natural competitor species category. Podospermum canum, Achillea setacea, Ranunculus acris and Poa pratensis are generalist species (stresstolerant). Trifolium resutum is specialist species, and Plantago schwarzenbergiana is rare specialist species (stress-tolerant). The other plants are weeds.

\section{Measurement results of carbon-dioxide emission, soil moisture and soil temperature in 2019}

Measurement of carbon-dioxide emission, soil moisture and soil temperature were done in the grazing season, the results are shown in Table 2.

Results of measurement Carbon-dioxide emission $\left(\mathrm{kg} \times \mathrm{m}^{-2} \times \mathrm{h}-{ }^{1}\right)$, soil moisture (\%), soil temperature $\left({ }^{\circ} \mathrm{C}\right)$ in 2019 (Karcag)

\begin{tabular}{|c|c|c|c|c|c|c|c|c|c|c|c|c|}
\hline \multirow{2}{*}{$\begin{array}{c}\text { Date/ } \\
\text { Treatment }\end{array}$} & \multicolumn{4}{|c|}{$\begin{array}{c}\text { Carbon-dioxide emission } \\
\left(\mathrm{kg} \times \mathrm{m}^{-2} \times \mathrm{h}^{-1}\right)\end{array}$} & \multicolumn{4}{|c|}{ Soil moisture (\%) } & \multicolumn{4}{|c|}{ Soil temperature $\left({ }^{\circ} \mathrm{C}\right)$} \\
\hline & $\mathbf{A} / \mathbf{M}(\mathbf{1})$ & $\mathbf{A} / \mathbf{E}(2)$ & A/Ö(3) & $\mathrm{A} / \mathrm{L}(\mathbf{4})$ & A/M(1) & $\mathbf{A} / \mathbf{E}(2)$ & A/Ö(3) & $\mathrm{A} / \mathrm{L}(\mathbf{4})$ & $\mathbf{A} / \mathbf{M}(\mathbf{1})$ & $\mathrm{A} / \mathbf{E}(2)$ & A/Ö(3) & $A / L(4)$ \\
\hline $28 \operatorname{Mar} 2019$ & 0.203 & 0.215 & 0.252 & 0.173 & 5.28 & 5.27 & 6.11 & 5.42 & 25.13 & 27.87 & 25.01 & 28.53 \\
\hline 10 Apr 2019 & 0.389 & 0.257 & 0.372 & 0.454 & 4.02 & 4.30 & 5.54 & 4.06 & 25.14 & 25.18 & 26.19 & 29.94 \\
\hline 25 Apr 2019 & 0.283 & 0.226 & 0.300 & 0.530 & 3.56 & 3.10 & 3.92 & 3.61 & 29.87 & 32.39 & 31.54 & 27.78 \\
\hline 08 May 2019 & 0.428 & 0.286 & 0.610 & 0.287 & 12.53 & 13.57 & 13.06 & 11.07 & 29.83 & 30.68 & 33.60 & 32.87 \\
\hline 23 May 2019 & 0.738 & 0.859 & 0.711 & 0.801 & 14.41 & 9.81 & 13.71 & 11.55 & 27.18 & 27.30 & 25.62 & 27.81 \\
\hline 6 June 2019 & 0.608 & 0.772 & 0.844 & 0.681 & 12.02 & 10.33 & 7.94 & 8.84 & 32.65 & 33.32 & 33.88 & 32.79 \\
\hline 19 June 2019 & 0.422 & 0.581 & 0.742 & 0.543 & 3.31 & 2.80 & 3.49 & 4.80 & 33.85 & 34.51 & 35.58 & 38.17 \\
\hline 4 July 2019 & 0.248 & 0.207 & 0.278 & 0.138 & 2.76 & 1.37 & 1.78 & 1.20 & 28.87 & 28.30 & 28.53 & 30.14 \\
\hline 22 July 2019 & 0.221 & 0.408 & 0.187 & 0.146 & 2.61 & 2.89 & 2.60 & 2.96 & 40.15 & 39.73 & 43.15 & 44.11 \\
\hline 5 Aug 2019 & 0.407 & 0.435 & 0.510 & 0.429 & 3.92 & 4.25 & 4.30 & 3.67 & 32.20 & 36.12 & 35.98 & 34.15 \\
\hline 15 Aug 2019 & 0.346 & 0.202 & 0.339 & 0.252 & 3.18 & 2.66 & 3.19 & 3.16 & 40.10 & 39.99 & 35.93 & 28.70 \\
\hline 29 Aug 2019 & 0.161 & 0.180 & 0.179 & 0.176 & 3.19 & 3.11 & 2.42 & 2.79 & 34.14 & 31.67 & 33.00 & 28.84 \\
\hline 11 Sept 2019 & 0.427 & 0.328 & 0.594 & 0.206 & 3.53 & 4.26 & 2.99 & 3.98 & 29.83 & & 31.70 & 29.47 \\
\hline 1 Oct 2019 & 0.194 & 0.313 & 0.264 & 0.177 & 4.10 & 2.71 & 5.42 & 2.12 & 29.59 & 29.17 & 29.92 & 31.23 \\
\hline Expected value & 0.36 & 0.38 & 0.44 & 0.36 & 5.60 & 5.03 & 5.46 & 4.95 & 31.32 & 31.80 & 32.12 & 31.75 \\
\hline Median & 0.37 & 0.30 & 0.36 & 0.27 & 3.74 & 3.68 & 4.11 & 3.83 & 29.85 & 31.18 & 32.35 & 30.04 \\
\hline Scatter & 0.1641 & 0.2167 & 0.2226 & 0.2168 & 4.0845 & 3.5843 & 3.7439 & 3.2279 & 4.6503 & 4.5294 & 4.9249 & 4.5812 \\
\hline Variance & 0.02693 & 0.0469 & 0.0495 & 0.0470 & 16.6837 & 12.8477 & 14.0168 & 10.4198 & 21.6261 & 20.5158 & 24.2552 & 20.9878 \\
\hline Kurtosis & 0.7622 & 0.8841 & -1.1767 & -0.4975 & 0.6955 & 1.3058 & 1.4044 & 0.5051 & 0.1880 & -0.4392 & 0.5622 & 3.3425 \\
\hline Skewness & 0.9491 & 1.3659 & 0.5272 & 0.8117 & 1.5289 & 1.4991 & 1.4944 & 1.2607 & 0.7408 & 0.6044 & 0.4576 & 1.8062 \\
\hline Minimum & 0.161 & 0.180 & 0.179 & 0.138 & 2.61 & 1.37 & 1.78 & 1.20 & 25.13 & 25.18 & 25.01 & 27.78 \\
\hline Maximum & 0.738 & 0.859 & 0.844 & 0.801 & 14.41 & 13.57 & 13.71 & 11.55 & 40.15 & 39.99 & 43.15 & 44.11 \\
\hline Summary & 5.08 & 5.27 & 6.18 & 4.99 & 78.42 & 70.43 & 76.47 & 69.23 & 438.53 & 445.18 & 449.63 & 444.53 \\
\hline Number of pieces & 14 & 14 & 14 & 14 & 14 & 14 & 14 & 14 & 14 & 14 & 14 & 14 \\
\hline
\end{tabular}

1: „Mulching” treatment, 2: „One-sided mowing” treatment, 3: „Ancient lawn” treatment, 4: „Grazing control” treatment.

The highest carbon-dioxide value was measured on $23^{\text {rd }}$ May $2019\left(0.859 \mathrm{~kg} \times \mathrm{m}^{-2} \times \mathrm{h}^{-1}\right)$ in ,One-sided mowing" treatment, while the lowest value was on $4^{\text {th }}$ July $2019\left(0.138 \mathrm{~kg} \times \mathrm{m}^{-2} \times \mathrm{h}^{-1}\right)$ on a „Grazing control” area. The highest soil moisture value was measured on $23^{\text {rd }}$ May $2019(14.41 \%)$ in „Mulching” treatment, while the lowest value was on $4^{\text {th }}$ July $2019(1.20 \%)$ on „Grazing control” area. The highest soil temperature was measured on $22^{\text {nd }}$ July $2019\left(44.11{ }^{\circ} \mathrm{C}\right)$ on
„Grazing control" area, while the lowest value was on $28^{\text {th }}$ March $2019\left(25.01^{\circ} \mathrm{C}\right)$ in ,Ancient lawn”.

Values on $11^{\text {th }}$ September 2019 are very important and require further measurements, because they can forecast the states caused by drought of climatic change on different utilized turfs. July in 2019 was droughty based on climatic index, while August was semi-desert. At the beginning of September $22.2 \mathrm{~mm}$ precipitation fell. In paradox way, there were higher values of soil moisture by „One-sided mowing” and „Grazing 
control" treatments than by „Ancient lawn” and „Mulching" treatments. It is possible that moor phytomass holds the small amount of precipitation falls after the longer dry period, so it cannot leak into the soil. By measuring carbon-dioxide emission, we found that there were higher values by „Mulching” and „Ancient lawn" treatments than on „One-sided mowing” and „Grazing control” areas. Our results were similar to those of Kovács (2014), Zsembeli et al. (2015) and Birkás (2017). On the areas covered with mulch and moor there is larger microbiological activity, because moor and mulch are degradable and get into the soil as organic material, which results higher carbon-dioxide emission even in drought if the soil moisture is not optimal for microbial activity. By measuring soil temperature, the values were higher by „Mulching” and „Ancient lawn" treatments than on „One-sided mowing” and „Grazing control” areas, which increase microbiological activity in the soil.

\section{CONCLUSION}

In our study, we found that succession process (shrub) has been increasing since the beginning of the survey (10 years) on the zero utilised area.
In 2019, because of droughty year non-covered areas were found with coenological investigation by Balázs in every parcel. Plants were classified into Social Behaviour Types by Borhidi and we concluded that natural competitors and stress tolerant species are in higher proportion in treatments than ruderal species, which signs the stability of the natural turf association. By measuring soil moisture, we found that the little precipitation falls after droughty period remains in mulch and moor, so by these treatments after a longer dry period smaller moisture value is expected. In similar cases (whose frequency will possibly increase) on parcels covered with moor phytomass the carbondioxide emission is the highest because of the extra degrading organic material. The confirm of this tendency requires further identifications.

\section{ACKNOWLEDGEMENT}

The study is the result of EFOP-3.6.2-16-201700001, ,Complex country economy and sustainability development research, service system in the Carpathian Basin" project.

\section{REFERENCES}

Bajnok, M.-Török, G.-Resch, R.-Buchgraber, K.-Tasi, J. (2011): A termőhely, a gyeptípus és az időjárás szerepe néhány gyep hozamának alakulásában a hasznosítás intenzitásának függvényében. Gyepgazdálkodási Közlemények. 2010/2011.13-18.

Bajor, Z.-Zimmermann, Z.-Szabó, G.-Fehér, Zs.-Járdi, I.-Lampert, R.-Kerény-Nagy, V.-Penksza, P.-L. Szabó, Zs.-Székely, Zs.Wichmann, B.-Penksza, K. (2016): Effect of conservation management practices on sand grassland vegetation in Budapest, Hungary. Applied Ecology and Environmental Research. 14 (3): 233-247.

Bakker, J.P.-Berendese, F. (1999): Constraints in the restoration of ecological diversity in grassland and heathland communities. Trends in Ecology and Evolution 14: 6368.

Balázs, F. (1949): A gyepek termésbecslése növénycönológia alapján. Agrártudományok. 1. 25-35.

Barcsák, Z.-Baskay-Tóth, B.-Prieger, K. (1978): Gyeptermesztés és hasznosítás. Mezőgazdasági Kiadó, Budapest. 32-103.

Bartha, S.-Szentes, Sz.-Horváth, A.-Házi, J.-Zimmermann, Z.Molnár, Cs.-Dancza, I.-Margóczi, K.-Pál, R.-Purger, D.Schmidt, D.-Óvári, M.-Komoly, C.-Sutyinszki, Zs.-Szabó, G.Csathó, A. I.-Juhász, M.-Penksza, K.-Molnár, Zs. (2014): Impact of mid-successional dominant species on the diversity and progress of succession in regenerating temperate grasslands. Applied Vegetation Science. 17(2): 201-213.

Baskay-Tóth, B. (1962): Legelő és rétművelés. Mezőgazdasági Kiadó, Budapest. 127-159.

Birkás, M. (2017): Földművelés és földhasználat. Mezőgazda Lap-és Könyvkiadó, Budapest. pp. 482.

Borhidi, A. (1993): A magyar flóra szociális magatartástípusa, természetességi és relatív ökológiai értékszámai. KTM-OTVHJPTE kiadványa, Pécs. pp. 93.
Brockway, D.G.-Gatewood, R.G.-Paris, R.B. (2006): Restoring fireas an ecological process in shortgrass prairie ecosystems: initial effects of prescribed burning during the dormant and growing seasons. Journal of Environmental Management. 65: 135162.

Czóbel, Sz.-Szirmai, O.-Németh, Z.-Gyuricza, Cs.-Házi, J.-Tóth, A.-Schellenberger, J.-Vasa, L.-Károly, P. (2012): Short-term effects of grazing exclusion on net ecosystem $\mathrm{CO}_{2}$ exchange and net primary production in a Pannonian sandy grassland. Notula Bot Horti Agrobo. 40: 67-72.

Da Ronche, F.-Ziliotto, U.-Scotton, M. (2002): Floristic composition Masscio del Monte Grappa (NE Italy) pastures in relation with de utilisation intensity. Multi Function Grassland.

Dér, F.-Marton, I.-Németh, T.-Pásztor, L.-Szabó, J. (2003): Termőhelyi minősítés a gyepgazdálkodásban. In: Gaál, Z.-Máté, F.-Tóth, G. (2003): Földminősítés és földhasználati információ. Keszthely, 2003. december 11-12., Országos konferencia kiadványa. 5; pp. 125-130.

Erdős, L.-Bátori, Z.-Tölgyesi, Cs.-Körmöczi, L. (2014a): The moving split window (MSW) analysis in vegetation science - an overview. Applied Ecology and Environmental Research. 12: 787805.

Erdős, L.-Cserhalmi, D.-Bátori, Z.-Kiss, T.-Morschhauser, T.Benyhe, B.-Dénes, A. (2013): Shrub encroachment in a woodedsteppe mosaic: combining GIS methods with landscape historical analysis. Applied Ecology and Environmental Research. 11: 371384.

Erdős, L.-Tölgyesi, Cs.-Dénes, A.-Darányi, N.-Fodor, A.-Bátori, Z.-Tolnay, D. (2014b): Comparative analysis of the natural and semi-natural plant communities of Mt Nagy and other parts of the Villány Mts (south Hungary). Thaiszia Journal of Botany. 24: $1-21$. 
Ferrer, C.-Broca, A. (1999): El bonimio agricultura-ganaderia en los ecosistemas mediterráneos. Actas de la XXXIX. Reunion Cientifica de la Sociedad Espanola para el Estudio de los Pastos. 309-344.

Halász, A. (2018): A gyephasznositás hatása a gyep hozamára. Értékálló Aranykorona. 18(3): 24-25.

Halász, A.-Tasi， J.-Bajnok，M.-Szabo, F.-Orosz， Sz. (2018) Climate sensitivity of Hungarian grasslands. In: Horan, B.Hennessy, D.-O'Donovan, M.-Kennedy, E.-McCarthy, B.Finn, J. A.-O'Brien, B. (szerk.) (2018): Sustainable meat and milk production from grasslands. Cork, Írország. Wageningen Academic Publishers. 598-600.

Hansson, M.-Fogelfors, H. (2000): Management of a semi-natura grassland, results from a'5-year-old experiment in southern Sweden. Journal of Vegetation Science. 11: 31-38.

Harcsa, M.-Kulin, B.Gy.-Sallai, A.-Penksza, K.-Szemán, L. (2011) Intenzív gyepek gyomosodási viszonyai a tápanyag utánpótlás megszüntetése után. Növényvédelem. 47 (7): 321-326.

Harcsa, M.-Szemán, L.-Bajnok, M.-Penksza, K. (2008): Extenzív gyeptermesztés hatása a telepített gyepalkotó fajok állományösszetételére. I. Gödöllői Állattenyésztési Tudományos Napok. Gödöllö, 2008. április 11-12. AWETH. 4(2): 761-768.

Harcsa， M.-Szemán, L.-Penszka， K. (2009): Telepített gyep szukcessziós folyamata az intenzív termesztéstechnológia felhagyása után. Tájökológiai Lapok. 7(2): 409-416.

Harcsa, M. (2009): Stress effects of extensive and intensive nutrient supply on grassland coenosys. Cereal Research Communications. 37: 269-272.

Isselstein, J.-Jeangros, B.-Pavlu, V. (2005): Agronomic aspects of biodiversity targeted management of temperate grasslands in Europe. Agricultural Research. 3: 139-151.

Kahmen, S.-Poschlod, P.-Schreiber, K.F. (2002): Conservation management of calcareous grasslands. Boilogical Conservation 104: 319-324.

Kovács, Gy. (2014): Mezőgazdasági hasznosítású talajok széndioxidemissziójának vizsgálata Karcag térségében. $\mathrm{PhD}$ értekezés, Debrecen. 1-145.

Kozák, L. (2011): Élőhely-kezelés. Debreceni Egyetem. Agrár-és Gazdálkodástudományok Centruma. pp. 86.

Központi Statisztikai Hivatal (2018): 4.1.4. Földhasználat művelési ágak és gazdaságcsoportok szerint (1990-). www.ksh.hu

Magyar, I.E. (2009): gyógynövényes gyep telepíthetősége. Gyepgazdálkodási módszerek növényállományra gyakorolt hatásának értékelése. PhD értekezés, Gödöllö. pp. 193.

Margóczi, K. (2003): Természetközeli gyepek regenerációja és restaurációja. Természetes állattartás, Debrecen. 6: 50-56.

Molnár, Zs.-Csízi, I. (2015): Természetkímélő gazdálkodás szikeseken. Magyarországi Természetvédelmi Közalapítvány Hálózat, Csákvár - MTA ÖK ÖBI, Vácrátót. pp. 91.

Nagy, G. (2001): A gyephasználat és a vidékfejlesztés összefüggései. Gyepgazdálkodásunk helyzete és kilátásai. DGyN 17. Debrecen. pp. 24-25.

Ónodi, G.-Kertész, M.-Botta-Dukát, Z.-Altbacker, V. (2008): Grazing effects on vedetation composition and on the spread of fire on open sand grasslands. Arid Land Research and Management. 22: 273-285.

Pápay, G. (2016): Cserjeirtás után magára hagyott, legeltetett és kaszált gyepterületek vegetációjának összehasonlító elemzése parádóhutai (Mátra) mintaterületen. Gyepgazdálkodási Közlemények. 14: 2 37-48.
Penksza, K.-Fehér, Á.-Saláta, D.-Pápay, G.-S-Falusi, E.-KerényiNagy, V.-Szabó, G.-Wichmann, B.-Szemethy, L.-Katona, K. (2016): Gyepregeneráció és vadhatás vizsgálata cserjeirtás után parádóhutai (Mátra) mintaterületen. Gyepgazdálkodási Közlemények. 14(1): 31-41.

Penksza, K.-Pápay, G.-Házi, J.-Tóth, A.-Saláta-Falusi, E.-Saláta, D.-Kerényi-Nagy, V.-Wichmann, B. (2015): Gyepregeneráció erdőirtással kialakított gyepekben mátrai (Fallóskút) mintaterületeken. Gyepgazdálkodási Közlemények. 13(1-2): 31-44.

Perevolotsky, A.-Seligman, N.G. (1998): Role of grazing in Mediterranean rangeland ecosystems. Bioscience. 48: 10071017.

Ryser, P.-Langenauer, R.-Gigon, A. (1995): Species richness and vegetation structure in a limestone grassland after 15 year management with six biomass removal regimes. Folia geobotanica-Phytotaxonomica. 30: 157-167.

Stefler, J.-Nagy, G.-Vinczeffy, I. (2000): Különbözö adottságú gyepek hasznosíthatósága húsmarhatartással. Állattenyésztés és Takarmányozás. 49/6: 495-496.

Szabó, G.-Zimmermann, Z.-Szentes, Sz.-Sutyinszki, Zs.-Penksza, K. (2010): Természetvédelmi és gyepgazdálkodási vizsgálatok a Dinnyési-Fertö gyepeiben. Gyepgazdálkodási Közlemények. 8: 31-38.

Szemán, L. (2006). Gyepgazdálkodási ismeretek. Egyetemi jegyzet, Gödöllö. pp. 89.

Szente, K.-Nagy, Z.-Tuba, Z. (1998): Enhanced water use efficiency in dry loess grassland species grown at elevated air $\mathrm{CO}_{2}$ concentration. Photosynthetica. 35: 637-640.

Szentes, Sz.-Sutyinszki, Zs.-Zimmermann, Z.-Szabó, G.-Járdi, I.Házi， J.-Penksza， K.-Bartha， S. (2011): A fenyérfü (Bothriochloa ischaemum (L.) Keng 1936) gyep bétadiverzitására gyakorolt hatásainak vizsgálata és értékelése mikrocönológiai módszerekkel. Tájökológiai Lapok. 9(2): 463475.

Szentes, Sz.-Sutyinszki, Zs.-Szabó, G.-Zimmermann, Z.-Házi, J.Wichmann, B.-Hufnágel, L.-Penksza, K.-Bartha, S. (2012): Grazed Pannonian grassland beta-diversity changes due to $\mathrm{C} 4$ yellow bluestem. Cent. Eur. J. Biol. 7(6): 1055-1065.

Tóth, Cs.-Nyakas, A.-Nagy, G.-Nan, Z.B. (2002): A comparison of two arid steppe vegetations from different geograpgical regions. Multifunction Grasslands. La Rochelle. 170-171.

Török, P.-Arany, I.-Prommer, M.-Valkó, O.-Balogh, A.-Vida, E.Tóthmérész, B.-Matus, G. (2007): Újrakezdett kezelés hatása fokozottan védett kékperjés láprét fitomasszájára, faj-és virággazdagságára. Természetvédelmi Közlemények. 13: 187198.

Vinczeffy, I. (1993): Legelő- és gyepgazdálkodás. Mezőgazda Kiadó, Budapest. pp. 223-242.

Williems, J.H.-Bik, L.P.M. (1998): Restoration of high species density in calcareous grassland: the role of seed rain and soil seed bank. Appl. Veg. Sci. 1: 91-100.

Zsembeli, J.-Szücs, L.-Tuba, G.-Czimbalmos, R. (2015): Nedvességtakarékos talajmúvelési rendszer fejlesztése Karcagon. In: Madarász, B. (2015): Környezetkímélő talajművelési rendszerek Magyarországon. MTA CSFK FTI, Budapest. pp. 122-133. 
